\title{
Detailed Microstructure Characterizations of BEOL Cu Interconnects
}

Bianzhu Fu ${ }^{1,2}$, Michael A. Gribelyuk ${ }^{3}$ and Seungman Choi $^{4}$

1. CCA Characterization, GLOBALFOUNDRIES, 400 Stone Break Road Extension, Malta, NY 12020

2. Currently at: Entegris Inc., 7 Commerce Dr., Danbury, CT 06810

3. ATD Characterization, GLOBALFOUNDRIES, 400 Stone Break Road Extension, Malta, NY 12020

4. Quality and Reliability Assurance, GLOBALFOUNDRIES, 400 Stone Break Road Extension, Malta, NY 12020

$\mathrm{Cu}$ interconnects in the back-end-of-line (BEOL) of semiconductor integrated chips were first introduced by IBM in 1997 through dual-damascene $\mathrm{Cu}$ filling and subsequent chemical-mechanical planarization (CMP) process. Due to its higher melting point, superior electrical and thermal conductivity, $\mathrm{Cu}$ wires usually can withstand a much higher current density than aluminum without reliability issue caused by electromigration (EM) [1]. However, as the semiconductor devices continue to shrink in size, the EM lifetime degrades as well due to the geometrical scaling of the critical void size [2]. Cu grain structure and texture are major factors, which affect the EM lifetime [3]. This makes detailed characterization of the $\mathrm{Cu}$ microstructure essential.

X-ray Diffraction (XRD) and Electron Backscatter Diffraction (EBSD), which is implemented in the scanning electron microscope (SEM), have been extensively applied to study the grain size and texture of bulk samples. However, they are generally not suitable to the study of the $\mathrm{Cu}$ interconnects in the semiconductor devices due to insufficient spatial resolution. Therefore, transmission electron microscopy (TEM) based method is often required to study the detailed microstructure of $\mathrm{Cu}$ interconnects. With the introduction of precession electron diffraction (PED) [4], microstructure analysis similar to the SEM-EBSD technique but with higher spatial resolution becomes possible. In this study, TEM-PED techniques are utilized to perform detailed microstructure analysis of $\mathrm{Cu}$ interconnects related failure analysis and reliability analysis.

Figure 1 is an example of a failure analysis. While conventional physical analysis in Fig. 1(a) and Fig. 1(b) shows the $\mathrm{Cu}$ bump and also a void in the metal line, a more detailed $\mathrm{Cu}$ microstructure analysis shows that at the Cu bump location, several small grains are present (Fig. 1(c)). This indicates, that the Cu bump is not due to an abnormal grain growth, but rather due to recrystallization in the later process.

Figure 2 is a study of the effect of metal line width on $\mathrm{Cu} \mathrm{EM}$ life. It is interesting to find that $\mathrm{Cu} \mathrm{EM}$ life increases with metal line width, but saturates at $96 \mathrm{~nm}$. A detailed microstructure analysis of the $\mathrm{Cu}$ line grain size shows a similar trend: grain size increases but almost saturates at $96 \mathrm{~nm}$. Also, texture analysis shows that as the metal width increases, more (111) textured $\mathrm{Cu}$ film is formed in the growth direction. This kind of microstructural information is important to understand the EM phenomenon in modern semiconductor devices and improve BEOL Cu reliability.

\section{References:}

[1] J. Lienig, "Introduction to Electromigration-Aware Physical Design", Proc. of the Int. Symposium on Physical Design (ISPD) 2006, p. 39.

[2] C. K. Hu, L. Gignac, and R. Rosenberg, Microelectron. Reliab. 46 (2006), p. 213. 
[3] Changsup Ryu, Alvin L. S. Loke, Takeshi Nogami and S. Simon Wong, "Effect of Texture on the Electromigration of CVD Copper”, Proceedings of IEEE International Reliability Physics Symposium 1997, p. 201.

[4] R.Vincent et al, Ultramicroscopy 53 (1994), p. 271.

[5] The authors acknowledge Brian Popielarski, Craig Petrask, Sylvester Amoah, Albert Amann III, and Michael D’Aniello for preparing TEM samples.
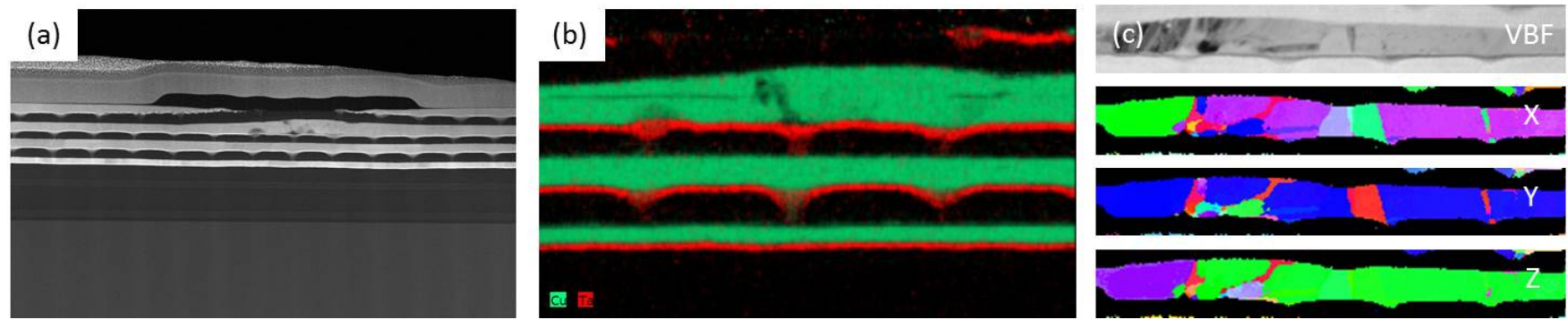

Figure 1. STEM image (a) and EDS elemental map (b) of the defect location, (c) microstructure characterization of the defect: VBF is virtual bright field image, $X$ is the direction parallel to the metal line, $\mathrm{Y}$ is the $\mathrm{Cu}$ line growth direction, and $\mathrm{Z}$ is the out-of-plane direction.
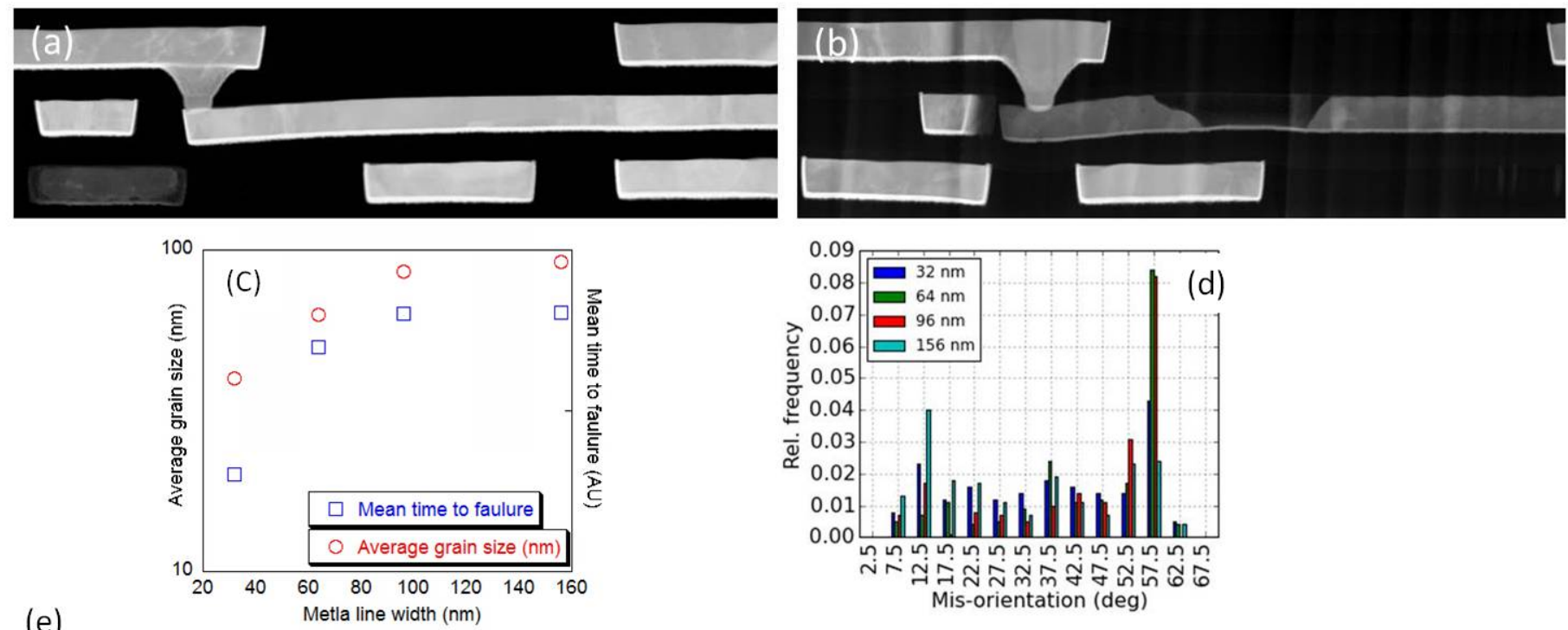

(e)

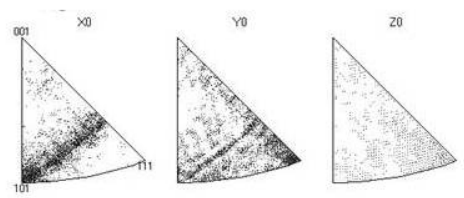

$32 \mathrm{~nm}$
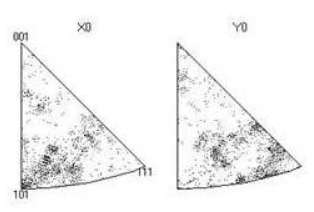

$64 \mathrm{~nm}$
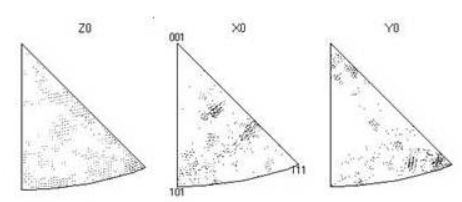

$96 \mathrm{~nm}$
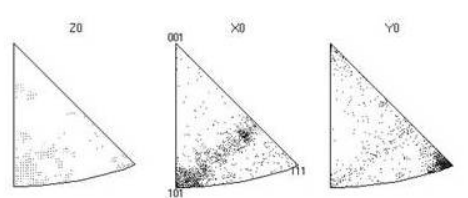

$156 \mathrm{~nm}$

Figure 2. (a) STEM image of M2 with $156 \mathrm{~nm}$ width (a) and $32 \mathrm{~nm}$ width (b), (c) summary of M2 grain size and mean time to failure in log scale and (d) grain boundary mis-orientations with various metal width, (e) inverse pole figure of M2 with various metal width. 